$$
\begin{aligned}
& 165-21665 \\
& 1 \mathrm{~m} x-55200
\end{aligned}
$$

\title{
RADIO EMISSION FROM SHOCK WAVES AND TYPE II SOLAR OUTBURSTS
}

FEBRUARY 1965

GODDARD SPACE FLIGHT CENTER GREENBELT, MARYLAND 
$X-641-65-68$

\section{RADIO EMISSION FROM SHOCK WAVES \\ AND TYPE II SOLAR OUTBURSTS}

by

Derek A. Tidman

February 1965

NASA-Goddard Space Flight Center

Greenbelt, Maryland 


\title{
RADIO EMISSION FROM SHOCK WAVES \\ AND TYPE II SOLAR OUTBURSTS
}

\author{
by \\ Derek A. Tidman* \\ NASA-Goddard Space Flight Center \\ Greenbelt, Maryland
}

\begin{abstract}
A model for Type II solar radio outbursts is discussed. The radiation is hypothesized to originate in the process of collective bremsstrahlung emitted by non-thermal electron and ion density fluctuations in a plasma containing a flux of energetic electrons. The source of radiation is assumed to be a collisionless plasma shock wave rising through the solar corona. It is also suggested that the collisionless bow shocks of the earth and planets in the solar wind might be similar but weaker sources of low frequency emission with a similar twoharmonic structure to Type II emission.
\end{abstract}

\footnotetext{
*Permanent address: Institute for Fluid Dynamics and Applied Mathematics, University of Maryland, College Park, Maryland.
} 


\title{
RADIO EMISSION FROM SHOCK WAVES \\ AND TYPE II SOLAR OUTBURSTS
}

\author{
by \\ Derek A. Tidman \\ NASA-Goddard Space Flight Center \\ Greenbelt, Maryland
}

\section{INTRODUCTION}

In a previous paper ${ }^{1}$ we calculated the bremsstrahlung emitted from thermal plasmas which co-exist with a flux of energetic (suprathermal) electrons. It was found that under some circumstances the radiation emitted from such plasmas can be greatly increased compared to the emission from a Maxwellian plasma with no energetic particles present. The enhanced emission occurs at the fundamental and second harmonic of the electron plasma frequency.

The object of this paper is to discuss a model for Type II solar radio outbursts in terms of the above radiation process. It has been suggested by several authors (for a review of the literature see reference 2) that this radiation is probably produced by longitudinal plasma oscillations which are excited by some mechanism associated with a shock wave rising through the solar corona. The longitudinal waves then convert part of their energy $3,4,5$ into electromagnetic radiation with a frequency $\omega_{\mathrm{e}}$ by scattering on density fluctuations in the corona, and part into radiation at $2 \omega_{\mathrm{e}}$ by scattering of longitudinal waves by each other $\left(\omega_{e}\right.$ is the electron plasma frequency $\left(4 \pi \mathrm{n}_{0} \mathrm{e}^{2 / m}\right)^{1 / 2}$ ).

The present model follows essentially along these same lines with the new feature that we are able to calculate quantitatively the bremsstrahlung from an assumed distribution function for the suprathermal electrons in the shocked plasma and point out the importance of Landau damping of the longitudinal waves by the suprathermal electrons. In the absence of adequate theories of collisionless plasma shock waves to guide us, we also assume that the shock wave in the corona has some of the structural features experimentally observed to characterize the earth's bow shock wave in the solar wind. ${ }^{6}$

One can visualize the energetic electrons behind the shock front as exciting Cerenkov electron plasma waves which then, through scattering from each other and by ion waves, convert part of their energy into electromagnetic radiation. 
We demonstrated previously, ${ }^{1}$ however, that the emission intensity is very sensitive to the velocity dependence of the distribution function, $f(\underline{v})$, in the high energy tail ( $\mathrm{f}$ includes both thermal and suprathermal electrons). This is because the Cerenkov electron plasma oscillations are also re-absorbed through Landau damping by the energetic electrons as well as emitted by them. This leads us to suggest a distribution function of a certain type for the energetic electrons in the shock wave (see Figure 3), namely one in which there is a gap in velocities between the thermal and energetic electrons. Such distributions are highly radiative (Note since $f=f(|\underline{v}|)$ they are also stable). In contrast, distributions which have high energy tails on them (representing a flux of energetic electrons in the medium) which merge continuously with the "thermal" particles, emit very little

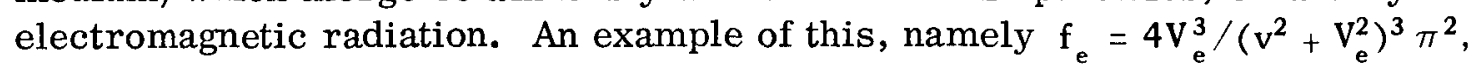
was treated in reference 1.

We shall next summarize briefly some of the observed properties of Type II outbursts. It is then also suggested that the earth's collisionless bow shock might emit electromagnetic radiation with a similar structure to Type $\Pi$ solar emission, but at lower frequencies ( $\mathrm{f}=\omega_{\mathrm{e}} / 2 \pi \simeq 20-300 \mathrm{kc} . / \mathrm{sec}$.). This radiation would only be detectable outside the magnetopause.

\section{CHARACTERISTICS OF TYPE II OUTBURSTS}

The data in this and the following section is taken from several sources. They include a review of solar radio emission by Roberts, ${ }^{7}$ the chapter by Pawsey and Smerd ${ }^{8}$ in "The Sun," papers by Maxwell and Thompson, 9 and more recent reviews of the literature by Wild, Smerd, and Weiss, ${ }^{2}$ and by Boyd. ${ }^{10}$

Type II, or "slow-drift" radio bursts, appear on the average about 6 minutes after the commencement of a solar flare and probably originate above about $10^{5} \mathrm{~km}$. over the photosphere. They are unpolarized and a large outburst ${ }^{9}$ radiates a total energy integrated over frequency and time of order $10^{22}$ ergs. This is as if an area of $10^{20} \mathrm{~cm}^{2}$ radiated like a black body at a temperature of $10^{13}$, i.e., they are a factor of about $10^{7}$ times more intense than the normal solar thermal emission from the above area.

The duration of these bursts is approximately 10 minutes, and their frequencies drift from high to low frequencies, which is interpreted as due to the radio source moving up through the solar corona at about 1 to $1.510^{8} \mathrm{~cm} / \mathrm{sec}$. Their velocity is deduced from the frequency drift by assuming that the frequencies observed correspond to the local plasma frequency at the source, which in turn depends on knowing the electron density in the region. 
Independent measurements of the height of the radio source in the corona using a swept-frequency interferometer ${ }^{2}$ leads to electron densities, $\mathrm{n}_{0}$, at the source which are of order 10 times the accepted values for the quiet corona. The deduced variation of $n_{0}$ with height agrees closely with those deduced optically by Newkirk ${ }^{11}$ for typical coronal streamers. The source is thus probably propagating out along a coronal streamer. (See Figure 1.)

One of the most puzzling features of these radio outbursts is the existence in most cases $(80 \%)$ of a strong second harmonic in the spectrum. Occasionally the second harmonic may be even stronger than the first, and yet there is no evidence of higher harmonics, even though they would have been visible in Maxwell and Thompson's work ${ }^{9}$ at an intensity of $20 \mathrm{~dB}$ lower than the fundamental or second harmonic. The maximum starting frequency, $f$, of the fundamental rarely exceeds $175 \mathrm{Mc}$. and often drifts to below $25 \mathrm{Mc}$. (It should be noted that most experimental authors quote frequencies in terms of the frequency $f=\omega_{e} / 2 \pi$ ).

The two harmonics sometimes have a similar structure, i.e., variations in intensity or line breadth occur simultaneously in both harmonics. They also sometimes both split into two sub-bands with a splitting frequency small compared to $\omega_{e}$. This latter effect is generally thought to have its origin in coronal

$$
f=\frac{\omega}{2 \pi}=9 \cdot 10^{-3} \sqrt{n_{0}} M c / s e c .
$$

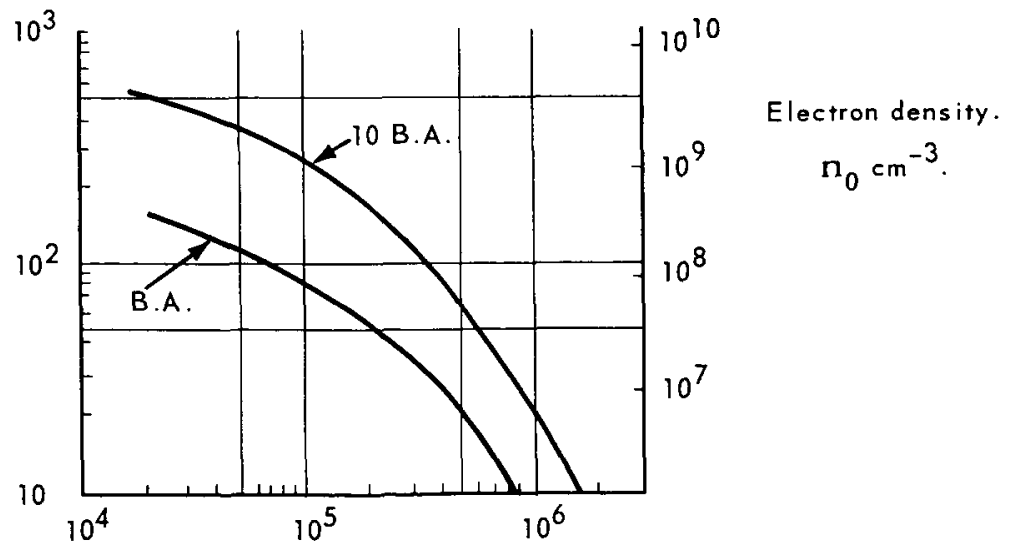

$$
\text { Height (km.) above photosphere. }
$$

Figure 1-Plot taken from Maxwell and Thompson (Ref. 9) of plasma frequency and electron density as functions of height above the photosphere. The lower curve is based on the Baumbach-Allen (B.A.) density distribution for the undisturbed corona, and the upper curve $(10 \times$ B.A. $)$ are values for a coronal streamer along which a Type II burst propagates. 
magnetic fields. Occasionally a "herring bone" effect is also observed, ${ }^{12}$ i.e., small fast drift bursts (Type III's) accompany each of the harmonics.

Another feature recently observed in some events is that the second harmonic appears to originate from deeper in the corona than the fundamental. ${ }^{13}$ Smerd, Wild, and Sheridan ${ }^{14}$ suggest that this is due to refraction of that part of the second harmonic radiation which is emitted down into deeper regions of the solar atmosphere. Additional refraction effects which may also play a role are due to the radiation traversing the irregular shock front and in leaving the coronal streamer and entering the quiescent corona.

\section{MODEL FOR TYPE II OUTBURSTS}

Suppose we assume that in the course of a solar flare a shock wave is generated by the upward expansion of the hot flare plasma into the solar corona. In the absence of adequate theories to guide us we shall assume that the collisionless shock wave is similar in structure to the bow shock wave which has been experimentally observed to be generated by the obstruction the earth's magnetic field presents to the "super-Alfvènic" solar wind. 6 Thus the shock transition is likely to be turbulent in nature and to propagate at some arbitrary angle to any undisturbed relatively ordered coronal magnetic field which may exist ahead of the wave (see Figure 2). We note that the shock velocity (of order $10^{8} \mathrm{~cm} / \mathrm{sec}$ ) is considerably greater than the Alfvèn speed, $B / \sqrt{4 \pi \mathrm{Mn}_{0}}$, for the range of densities and field strengths involved at the source of emission in the corona. $\left(\mathrm{B} \cong 0(1 \text { gauss }) \text { and } \mathrm{n}_{0} \cong 10^{6}-10^{8} \mathrm{~cm}^{-3}\right)^{*}$

Now in the turbulent region behind the collisionless bow shock wave of the earth, fluxes of energetic electrons are observed. ${ }^{15}$ The mechanism by which they are energized in or behind the shock front is not yet clear, although it is most plausible that it involves a stochastic process ${ }^{16}$ in the plasma turbulence behind the front of the wave. We assume that in the collisionless shock propagating up through the coronal plasma, a similar condition of disordered plasma and magnetic field co-existing with a flux of suprathermal electrons exists. On the basis of these assumptions we shall apply our bremsstrahlung calculations to see how many of the spectral characteristics of Type $\Pi$ 's we can explain.

\footnotetext{
*The magnetic field strength in the outer corona is not well known. In reference (9) it is pointed out that if the band splitting in Type II emission is interpreted as a gyromagnetic effect analogous to Zeeman splitting, the following field strengths are obtained: 1.5 gauss at $8.10^{5} \mathrm{~km}$. above the photosphere, 3 gauss at $4.10^{5} \mathrm{~km}$., and 8.5 gauss at $3.10^{5} \mathrm{~km}$.
} 


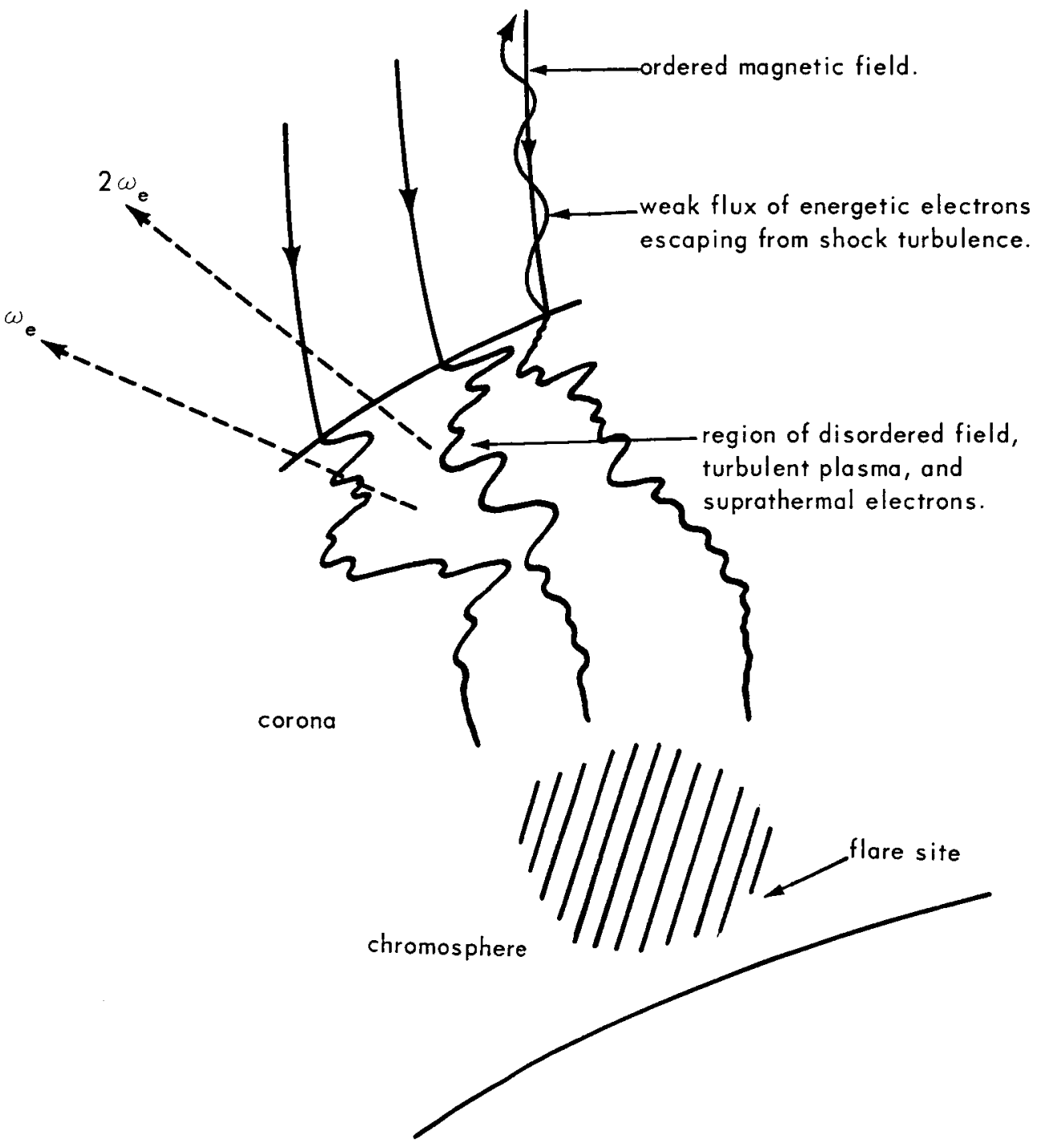

Figure 2-ldealized sketch of a model for a collisionless shock wave in the coronal plasma above a solar flare (assuming similar characteristics to the earth's bow shock)

The turbulent magnetic field behind the shock front will serve both to confine the energetic electrons to that region, and through scattering constrain the velocity distribution for the suprathermal electrons to be approximately isotropic. Although some energetic electrons are likely to escape into the ordered field ahead of the shock, they will rapidly travel upwards along the field lines and thus their intensity ahead of the shock should be much less than that behind the shock front. 
Previously we calculated the collective bremsstrahlung ${ }^{1}$ emitted from plasmas containing energetic particle fluxes, using several distribution functions. For the present application we shall assume that the plasma behind the shock front in the rest frame of the shock can be represented by the following distributions, $f_{i}$ and $f_{e}$ for the ions and electrons,

$$
\begin{gathered}
f_{i}(\underline{v})=\frac{1}{\left(2 \pi V_{i}^{2}\right)^{3 / 2}} \exp \left(-\frac{v^{2}}{2 V_{i}^{2}}\right) \\
f_{e}(\underline{v})=\frac{\beta}{\left(2 \pi V_{e}^{2}\right)^{3 / 2}} \exp \left(-\frac{v^{2}}{2 v_{e}^{2}}\right)+(1-\beta) I_{s}\left(|\underline{v}|-V_{E}\right) g(|\underline{v}|),
\end{gathered}
$$

where $\mathrm{I}_{\mathrm{s}}=0$ if $|\underline{\mathrm{v}}|<\mathrm{V}_{\mathrm{E}}$ and $=1$ otherwise. $\mathrm{V}_{\mathrm{e}}$ and $\mathrm{V}_{\mathrm{i}}$ are the electron and ion thermal velocities, $\mathrm{V}_{\mathrm{e}}=(\mathrm{KT} / \mathrm{m})^{1 / 2}=(\mathrm{M} / \mathrm{m})^{1 / 2} \mathrm{~V}_{\mathrm{i}}$. We also choose $\exp \left(-V_{E}^{2} / 2 V_{e}^{2}\right) \ll(1-\beta) \ll \beta \cong 1$ and $V_{E}>$ several $-V_{e}$ (see Figure 3). Note the distributions are all normalized to unity, $\int_{-\infty}^{\infty} \mathrm{d} \underline{v} \mathrm{f}_{\mathrm{i}, \mathrm{e}}=1$, and the number density of the electrons and ions is $n_{0}$. The function $g(|\underline{v}|)$ represents the distribution for the suprathermal electrons.

Now $f_{e}(\underline{v})$ as given in (2) represents a Maxwellian gas of electrons coexisting with a tenuous isotropic flux of energetic electrons all of which have velocities $|\underline{v}|>V_{E}$. We found that distributions of this type are highly radiative 1 and give enhanced emission at $\omega_{\mathrm{e}}=\left(4 \pi \mathrm{n}_{0} \mathrm{e}^{2} / \mathrm{m}\right)^{1 / 2}$ and $2 \omega_{\mathrm{e}}$. The basic reason for this is due to the gap in velocities $v_{e}<|\underline{v}|<v_{E}$ which only the few Maxwellian tail particles occupy. Electrostatic (longitudinal) Cerenkov waves are emitted into the phase velocity range several- $\mathrm{V}_{\mathrm{e}}<\omega / \mathrm{k}<\mathrm{V}_{\mathrm{E}}$ by the energetic electrons, but only reabsorbed (Landau damped) by the few thermal particles far out on the Maxwellian tail. These longitudinal waves then scatter off of each other and emit electromagnetic radiation.

A distribution function of this type is expected if the acceleration mechanism that energizes a preferred few of the electrons in the plasma is very efficient in a velocity range $|\underline{v}|<V_{E}$, so that they rapidly get carried up into the range $|\underline{v}|>V_{E}$. 


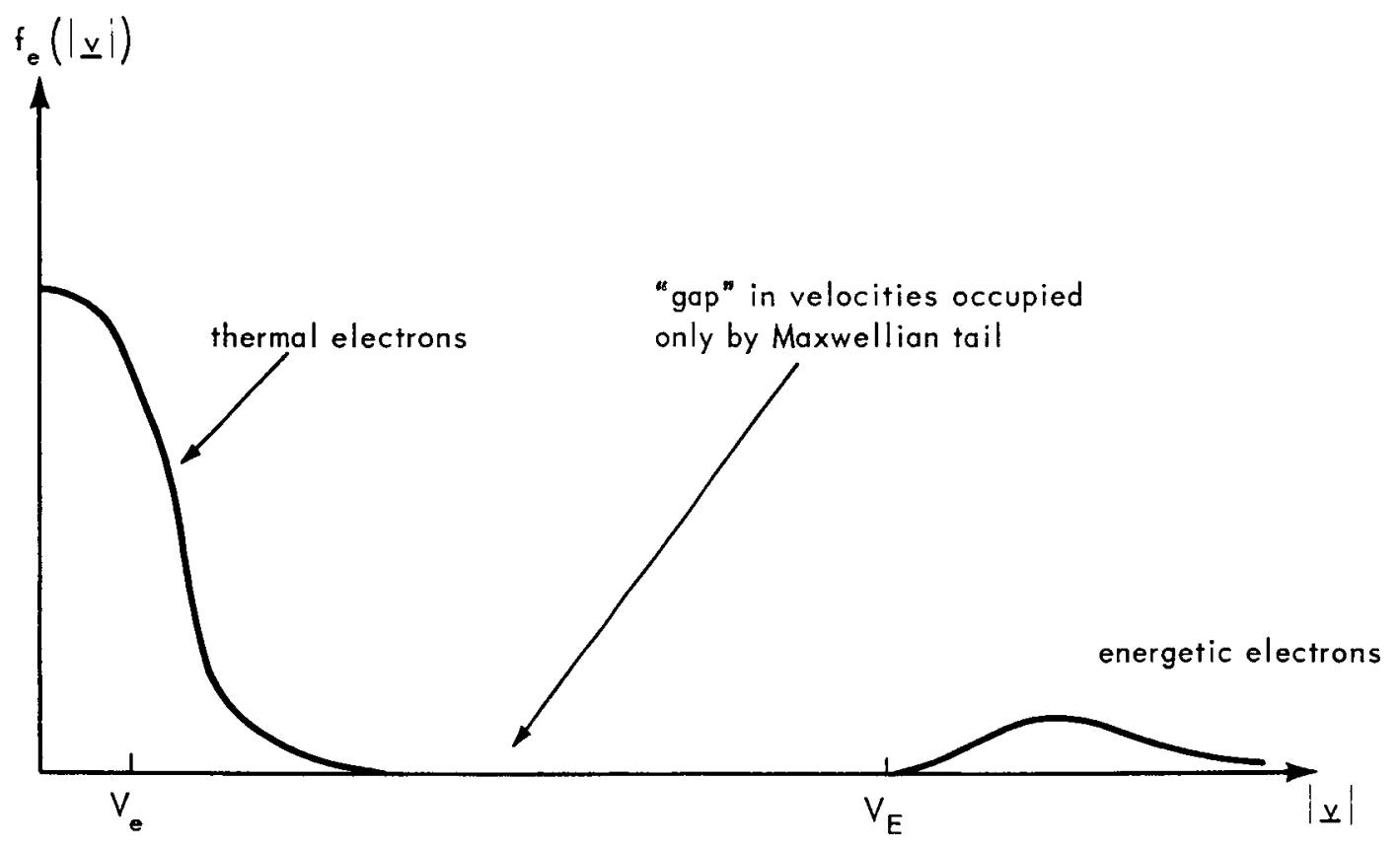

Figure 3-Schematic plot of $f_{e}\left(\left.\right|_{\underline{v}} \mid\right)$ as a function of $\left.\right|_{\underline{v}} \mid$ for $\beta<1$ as given by Equation (2)

On the other hand a resonance distribution, $f_{i, e}(|\underline{v}|)=4 V_{i, e}^{3} / \pi^{2}\left(v^{2}+V_{e, i}^{2}\right)^{3}$, for which the suprathermal electrons do contribute appreciably to Landau damping for all longitudinal waves of any phase velocity, gives little increase in the emission. ${ }^{1}$ This feature is characteristic of distributions for which the distribution of the "suprathermal" particles merges continuously in velocities with that of the "thermal" particles.

For the distributions (1) and (2) the first and second harmonic emission is (taking $C \cong 1 / 2$ in eq. (58) ref. 1 ).

$$
I_{\omega_{e}}(\text { i po - epo }) \cong \frac{(1-\beta)}{2 \pi \sqrt{3}} G J_{1}\left(\frac{\mathrm{mV}_{\mathrm{e}}^{2}}{\mathrm{n}_{0} \mathrm{~L}^{3}}\right) \omega_{\mathrm{e}}\left(\frac{\mathrm{V}_{\mathrm{e}}}{\mathrm{c}}\right)^{3} \frac{1}{\mathrm{~L}^{3}}\left(\frac{\mathrm{V}_{\mathrm{e}}}{\mathrm{V}_{\mathrm{E}}}\right)
$$

and

$$
\mathrm{ergs} / \mathrm{sec} \cdot / \mathrm{cm}^{3} \text {. }
$$

$$
\mathrm{I}_{2 \omega_{\mathrm{e}}}(\text { epo-epo }) \cong \frac{\pi \sqrt{3}(1-\beta)^{2}}{5} \mathrm{G}^{2} \mathrm{~J}_{2}\left(\frac{\mathrm{m} \mathrm{V}_{\mathrm{e}}^{2}}{\mathrm{n}_{0} \mathrm{~L}^{3}}\right) \omega_{\mathrm{e}}\left(\frac{\mathrm{V}_{\mathrm{e}}}{\mathrm{c}}\right)^{3} \frac{1}{\mathrm{~L}^{3}}\left(\frac{\mathrm{V}_{\mathrm{e}}}{\mathrm{V}_{\mathrm{E}}}\right)^{2}
$$


where,

$$
\begin{aligned}
& \mathrm{J}_{1} \cong\left(\frac{2}{\pi}\right)^{1 / 2}\left(\frac{\mathrm{V}_{\mathrm{e}}}{\mathrm{V}_{\mathrm{E}}}\right)^{5} \exp \left(\frac{\mathrm{V}_{\mathrm{E}}^{2}}{2 \mathrm{~V}_{\mathrm{e}}^{2}}\right) \\
& \mathrm{J}_{2} \cong \frac{1}{\pi}\left(\frac{\mathrm{V}_{\mathrm{e}}}{\mathrm{V}_{\mathrm{E}}}\right)^{3} \exp \left(\frac{\mathrm{V}_{\mathrm{E}}^{2}}{\mathrm{~V}_{\mathrm{e}}^{2}}\right)
\end{aligned}
$$

and

$$
G=\int_{v_{E}}^{\infty} v_{E} v d v g(v),
$$

and $\mathrm{L}$ is the Debye radius $\left(\mathrm{L}=\mathrm{V}_{\mathrm{e}} / \omega_{\mathrm{e}}\right)$. Note that the magnetic field has been neglected in calculating the above results. However although the coronal magnetic fields play a dominant role in the shock structure, and probably also in energizing the electrons represented by $\mathrm{g}(|\underline{\mathrm{v}}|)$, they are of secondary importance in computing the bremsstrahlung at frequencies $\omega \cong 0\left(\omega_{\mathrm{e}}\right)>>\Omega=\mathrm{e} . \mathrm{B} / \mathrm{mc}$. We are making calculations to see if the band splitting can be accounted for by inclusion of a "weak" magnetic field.

The emission intensities (3) and (4) were derived from convolutions involving the spectral densities $S_{e e}(\underline{k}, \omega)$ and $S_{i i}(\underline{k}, \omega)$ for the fluctuating number densities of the electrons and ions. For wave numbers $|\underline{k}|<k_{D}=L^{-1} \quad\left(k_{D}\right.$ is the Debye wave number), the spectral density $\mathrm{S}_{\mathrm{e} e}$ has resonances at $\omega \cong 0, \omega_{\mathrm{e}}$, and $S_{i i}$ has a resonance at $\omega \cong 0$. These resonances (see Figure 4) correspond to a spectrum of electron plasma oscillations (epo's) and ion plasma oscillations (ipo's) in the medium in the sense that they represent Fourier components of the spectral densities for which $\omega$ and $\mathrm{k}$ are approximately related as they would be for propagating Vlasov ion and electron plasma waves. Thus, roughly speaking, $I_{\omega_{e}}$ derives from epo-ipo scattering, and $I_{2 \omega_{e}}$ from epo-epo scattering. 
Now we note (Figure 4) that the presence of energetic electrons in the plasma enhances the epo components of $S_{e e}$ at $\omega \cong \omega_{e}$, but the fast electrons do not produce a corresponding excitation of the low frequency ipo modes at $\omega \cong \overline{0 \text {. (Com- }}$ pare $\mathrm{S}_{\mathrm{ee}}$ for $\beta=1, \beta<1$ in Figure 4). This results in a situation in which if the exponential factors $\exp \left(\mathrm{V}_{\mathrm{E}}^{2} / 2 \mathrm{~V}_{\mathrm{e}}^{2}\right)$ in (5) and (6) are large, then $I_{2 \omega_{e}}>I_{\omega_{e}}$. This is contrary to the observed fact that $I_{\omega_{e}}$ and $I_{2} \omega_{e}$ are of comparable orders of magnitude for a Type $I I$ outburst. In order to explain the observations we must find a mechanism which does enhance the low frequency mode excitations in the plasma, and the corresponding $I_{\omega_{e}}$.

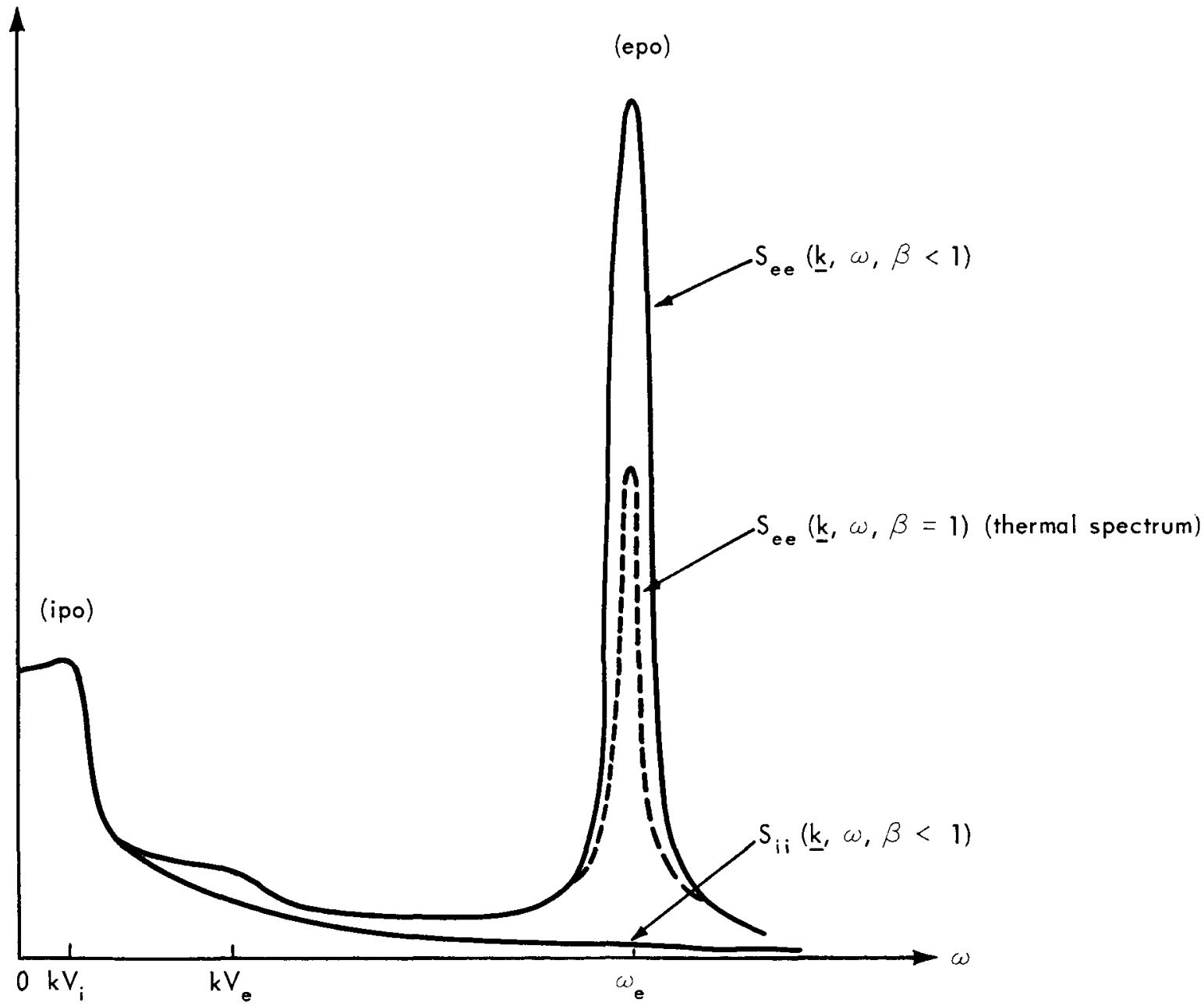

Figure 4-Schematic plot of the spectral densities $S_{e e}$ and $S_{i i}$ as functions of frequency for a fixed $k<k_{D}$ 
The most obvious source of low frequency density fluctuation is the shock turbulence itself. Now we are not yet in a position to calculate the spectral density for this turbulence - what is needed is a theory of turbulence for Vlasov plasmas and shock wave structure. However in the case of the earth's bow shock the magnetic field fluctuations are observed to be ${ }^{6}$ such that $|\Delta \underline{B}|$ is of $0(|\underline{B}|)$, and similarly $|\delta \rho| \cong 0(1)$ for the normalized density fluctuations and for wavelengths greater than an ion cyclotron radius. In the theory of reference 1 , however, the thermal fluctuations of a "homogeneous" plasma are such that $|\delta \rho| \cong 0$ (g) where $\mathrm{g}=1 / \mathrm{n}_{0} \mathrm{~L}^{3}$ is the plasma parameter. For the coronal plasma $\mathrm{g} \cong 10^{-8}$. Thus if the coronal shock wave is similar in structure to the earth's bow shock, then the spectral densities at frequencies $\omega<<\omega_{\mathrm{e}}$ will be increased by many orders of magnitude, perhaps by as much as $10^{8}$ in some wave numbers over those used in the derivation of (3). This suggests that it is capable of bringing $I_{\omega_{e}}$ up to the intensity level of $I_{2 \omega_{e}}$, but of course does not enable us to make a quantitative calculation. Since in our model $I_{2 \omega_{e}}$ most directly reflects the simpler properties of energetic electron fluxes in the shocked plasma, we shall in the rest of this section, examine the conditions under which $I_{2 \omega_{e}}$ is large enough to account for the observed power level at $2 \omega_{\mathrm{e}}$.

Considex for example the parameters (see Figure 1), $\mathrm{T} \cong 10^{6}{ }^{\circ} \mathrm{K}, \mathrm{V}_{\mathrm{e}} / \mathrm{c} \cong 10^{-2}$, $\mathrm{n}_{0} \cong 10^{8}, \mathrm{f} \cong 100 \mathrm{Mc} / \mathrm{sec}$, i.e., $\omega_{\mathrm{e}} \cong 600 \mathrm{Mc} / \mathrm{sec}$, , and for the Debye length, $\mathrm{L}=\mathrm{V}_{\mathrm{e}} / \omega_{\mathrm{e}} \cong .5 \mathrm{~cm}$. Using these in (4) we find,

$$
I_{2 \omega_{\mathrm{e}}} \cong 2 \cdot 10^{-14}(1-\beta)^{2} \mathrm{G}^{2}\left(\frac{\mathrm{V}_{\mathrm{e}}}{\mathrm{V}_{\mathrm{E}}}\right)^{5} \exp \left(\frac{\mathrm{V}_{\mathrm{E}}^{2}}{\mathrm{~V}_{\mathrm{e}}^{2}}\right) \text { ergs } / \mathrm{sec} . \mathrm{cm}^{3} \text {. }
$$

Various assumptions are now possible for the flux of suprathermal electrons behind the shock front. Thus, suppose we assume that the energy density of the suprathermal electrons is comparable to the thermal energy density of the plasma, i.e.,

$$
(1-\beta) \mathrm{n}_{0} \mathrm{~m} \mathrm{~V}_{\mathrm{E}}^{2} \cong \beta \mathrm{n}_{0} \mathrm{~m} \mathrm{~V}_{\mathrm{e}}^{2}
$$

Further, from the observed typical linear dimensions of solar flare plasma, we take the radiating volume of plasma behind the collisionless shock front to be $\cong 10^{29} \mathrm{~cm}^{3}$. Equation (8) now gives for the total emission (note $G \cong 0(1 / 4 \pi), \beta \cong 1$ ),

$$
I_{2 \omega_{\mathrm{e}}}^{\operatorname{total}} \cong 10^{13}\left(\frac{\mathrm{V}_{\mathrm{e}}}{\mathrm{V}_{\mathrm{E}}}\right)^{9} \exp \left(\frac{\mathrm{V}_{\mathrm{E}}^{2}}{\mathrm{~V}_{\mathrm{e}}^{2}}\right) \text { ergs/sec. }
$$


If the energetic electrons all lie in a velocity range $|\underline{v}|>V_{E} \cong 5.5 V_{e}$ (i.e., are in the energy range $>2.5 \mathrm{keV}$, the total emission becomes $I_{2 \omega_{\mathrm{e}}}^{\text {tota } 1 \cong 2.10^{19}}$ ergs/sec., which is the required power output to explain type II outbursts.

In reference 1 we noted two conditions for the applicability of (4) and we shall now show that these are satisfied.

\section{$\underline{\text { Landau Damping }}$}

The first condition is that there be an equilibrium established between the emission and re-absorption (Landau damping) of longitudinal (electrostatic) waves for the small wave-number part of the spectral density in the radiating volume of plasma. This is the case if the linear dimensions, $\mathrm{L}_{\mathrm{s}}$, of the shocked plasma is much larger than the propagation length $\left(\omega_{\mathrm{e}} / \mathrm{k}\right) \gamma_{\mathrm{L}}^{-1}(\mathrm{k})$ for a wave of phase velocity $\omega_{\mathbf{e}} / \mathbf{k} \cong \mathrm{V}_{\mathbf{E}} \cdot\left(\gamma_{\mathrm{L}}\right.$ is the Landau damping decrement). We require,

$$
\mathrm{L}_{\mathrm{s}} \gg \mathrm{L}\left[\mathrm{e}^{3 / 2} \sqrt{\frac{8}{\pi}}\left(\frac{\mathrm{V}_{\mathrm{e}}}{\mathrm{V}_{\mathrm{E}}}\right)^{2} \exp \left(\frac{\mathrm{V}_{\mathrm{E}}^{2}}{2 \mathrm{~V}_{\mathrm{e}}^{2}}\right)\right] \cong 3 \mathrm{~km} .
$$

for the parameters we have chosen. This condition is therefore satisfied.

\section{Collision Damping}

The second condition is that Landau damping be more important than collision (correlation) damping for electron plasma oscillations in the wave-number range considered. Equation (61b) of reference 1 gives this condition as,

$$
\left(\frac{V_{E}}{V_{e}}\right)^{3} \exp \left(-\frac{V_{E}^{2}}{2 V_{e}^{2}}\right)>\frac{1}{n_{0} L^{3}} \ln \left(n_{0} L^{3}\right)
$$

For the parameters we have chosen the left side of this equation is about 10 times the right side so that this condition is also met. We should point out in passing, however, that even if it were not satisfied, one would still expect greatly enhanced emission although our simple formula (4) would become modified. 


\section{LOW FREQUENCY EMISSION FROM THE EARTH'S BOW SHOCK}

For the purpose of estimating the emission in this case ${ }^{6}$ we assume the following parameters: $\mathrm{L}_{\mathrm{s}}^{3} \cong 10^{29} \mathrm{~cm}^{3}=$ radiating volume, $\mathrm{n}_{0} \cong 10, \mathrm{~T} \cong 10^{5}$, and $(1-\beta) \mathrm{n}_{0} \mathrm{mV} \mathrm{V}_{\mathrm{E}}^{2}=\alpha \beta \mathrm{mV}_{\mathrm{e}}^{2} \mathrm{n}_{0}$, where $\alpha$ is approximately the fraction of the thermal energy represented by the energy density of the suprathermal electrons. Thus $\mathrm{V}_{\mathrm{e}} \cong 1.210^{8} \mathrm{~cm} / \mathrm{sec}, \omega_{\mathrm{e}} \cong 1.510^{5}$ and $\mathrm{L} \cong 10^{3} \mathrm{~cm}$. Again we assume the electron acceleration mechanism carries a few thermal electrons rapidly up to velocities $|\underline{\mathrm{v}}| \geq \mathrm{V}_{\mathrm{E}}$, and that the region $|\underline{\mathrm{v}}|<\mathrm{V}_{\mathrm{E}}$ is occupied only by a few Maxwellian electrons. In the previous section we made a similar assumption for the plasma behind a coronal shock wave since only then could we get sufficient emission to explain the observed intensity of a Type $I$ burst. In the case of the region behind the earth's bow shock a similar energy gap between the thermal and suprathermal electrons may or may not exist, but we shall explore the hypothesis that it does.

The second harmonic emission then follows from (4) as,

$$
I_{2 \omega_{e}}^{\operatorname{total}} \cong 2.10^{-6} a^{2}\left(\frac{V_{e}}{V_{E}}\right)^{9} \exp \left(\frac{V_{E}^{2}}{V_{e}^{2}}\right) \text { ergs/sec. }
$$

This is now extremely sensitive to the ratio $\left(V_{e} / V_{E}\right)$. Thus if for example we take $\mathrm{V}_{\mathrm{E}} / \mathrm{V}_{\mathrm{e}} \cong 5.5$ as before, and assume $\alpha \cong 0(1)$, we find $\mathrm{I}_{2 \omega_{\mathrm{e}}} \cong 500 \mathrm{ergs} / \mathrm{sec}$. (at a steady frequency of $\mathrm{f}=2 \omega_{\mathrm{e}} / 2 \pi \cong 60 \mathrm{kc} . / \mathrm{sec}$.). The two conditions $(10)$ and (11) for the applicability of equation (4) are also satisfied for this choice.

Such radiation if it exists would have to be observed outside the magnetopause. Although its power level might be quite low and one would have to contend with possible low frequency background noise from the Sun and magnetosphere of the earth, we think it is worth looking for such emission - both from the earth and other planets. We cannot of course make a reliable prediction of the expected intensity for such a process due to the sensitivity of $I_{2 \omega_{e}}$ to the ratio $V_{E} / V_{e}$. However, such a pheonmenon if detected would give us a much closer look at the physical mechanisms involved in the corresponding solar Type II events.

\section{ACKNOWLEDGMENTS}

The author is indebted to Drs. T. H. Dupree, M. Krook, A. Maxwell, and N. F. Ness for many stimulating discussions. 


\section{REFERENCES}

1. D. A. Tidman and T. H. Dupree, Univ. of Md. Tech. Note, BN 384 (1965), (communicated to Phys. Rev.).

2. J. P. Wild, S. F. Smerd, and A. A. Weiss, Annual Review of Astronomy and Astrophysics, 1, 291 (1963).

3. V. L. Ginzburg and V. V. Zheleznyakov, Astron. Zh., 35, 694 (1958) [Engl. Transl., Soviet Astron. - AJ, 2, 653 (1958)]. See also M.A. Gintsburg, NASA draft translation ST-IS-PF-10141 of, Kosmich. Issledovaniya 2 , 65 (1964).

4. D. A. Tidman and G. Weiss, Phys. Fluids, 4, 703 (1961)., Phys. Fluids, 4, 866 (1961)., P.Sturrock, J. Nucl. Energy: Pt.C, 2, 158 (1961)., M. H. Cohen, Phys. Rev., 123, 711 (1961)., 126, 389 (1962)., 126, 398 (1962).

5. D. C. Montgomery and D. A. Tidman, Plasma Kinetic Theory, McGraw-Hill (1964).

6. N. F. Ness, C. S. Scearce and J. B. Seek, J. Geophys. Res. $\underline{69}, 3531$ (1964).

7. J. A. Roberts, Austral. J. Phys. 12, 327 (1959).

8. J. L. Pawsey and S. F. Smerd: The Sun (ed. Kuiper, 1953), Chapter 7.

9. A Maxwell and A. R. Thompson, Ap. J. 135, 138 (1962).

10. T. J. M. Boyd, Univ. of Md. Tech. Note BN-287 (1962). This contains a summary of the contents of 80 papers relevant to Type II bursts.

11. G. Newkirk, Astrophys. J., 133, 983 (1961).

12. J. A. Roberts, Austr. J. Phys., 12, 327 (1959).

13. A. A. Weiss, Austral. J. Phys., 17, 282 (1964).

14. S. F. Smerd, J. P. Wild, and K. V. Sheridan, Austral. J. Phys., 15, 180 (1962).

15. J. R. Jokipii and Leverett Davis, Jr., Phys. Rev. Lett. 13, 739 (1964)., C. Y. Fan, G. Gloeckler and J. A. Simpson, Phys. Rev. Lett. $\overline{13}, 149$ (1964)., K. A. Anderson, H. K. Harris and R. J. Paoli, Univ. of California at Berkeley, Space Sciences Lab. Rept. Series 5, No. 50 (1964)., J. W. Freeman, J. Van Allen, and L. J. Cahill, J. Geophys. Res. 68, 2121 (1963).

16. T. H. Stix, Phys. Fluids, 7, 1960 (1964). 